UAM Vol. 31 Pozлań 2004

\title{
ACERCA DE LOS PREDICADOS UNI- Y BIMODOACCIONALES (SOBRE LAS RELACIONES ENTRE LA ESTRUCTURA VALENCIAL Y LA AKTIONSART)
}

\begin{abstract}
Pawlak Aneta, Acerca de los predicados uni- y bimodoaccionales (sobre las relaciones entre la estructura valencial y la aktionsart) [About the 'uni- and bimodoaccional' predicates (about the relations between the valency's structure and the Aktionsart)]. Studia Romanica Posnaniensia, Adam Mickiewicz University Press, Poznań, vol. XXXI: 2004, pp. 417-422. ISBN 83-232-1353-4, ISSN 0137-2475.
\end{abstract}

This article is meant to present a new method of analysing the Aktionsart of predicates. There are a few factors which are taken into account: the valency's structure and the arguments' structure of the predicate; the nature of the subject and the direct object; and finally, the influence of some adverbial complements. All these elements ought to be thoroughly studied to determine a complete interpretation of the predicate's Aktionsart.

En nuestro trabajo abordamos dos diferentes campos de estudio sobre el predicado verbal. El primero está en relación con la categoría semántica de la Aktionsart' o modo de acción verbal, y el segundo, también de índole semántica, atañe a la estructura valencial del predicado verbal ${ }^{2}$.

Asimismo, vamos a tomar en consideración las características de los sustantivos que pueden desempeñar la función de complemento directo y la del sujeto al ser regidos por el núcleo del sintagma verbal. Además, nos detenemos brevemente en el análisis de los CCT que pueden acompañar, de manera adicional, al predicado dentro de la oración.

' Para más información sobre la Aktionsart véanse, por ejemplo, los trabajos de E. de Miguel (1999), Y. Morimoto (1998: 9-24), B. Hlibowicka-Węglarz (1998: 31-71).

${ }^{2}$ Al hablar de la estructura valencial semántica nos basamos en la teoría de Ch. Fillmore aludida por J.D. Apresjan (1974: 120-121). Además, remitimos al trabajo de Y. Morimoto (2001: 20-42) donde se presenta detalladamente la teoría de la Semántica Conceptual de Jackendoff que abarca la estructura léxico-conceptual, equivalente a la estructura valencial semántica y la estructura argumental del predicado. 
Nuestra propuesta, al partir de la coacción de los elementos gramaticales señalados supra, tiene por objetivo sugerir la posibilidad de un nuevo análisis concerniente a la Aktionsart ${ }^{3}$ de los predicados verbales de la lengua española. Este análisis consiste en establecer una jerarquía de los factores semántico-sintácticos que influyen en la determinación de las relaciones modoaccionales del predicado ${ }^{4}$.

El primer escalón de esta jerarquía, según nuestras observaciones, debe corresponder a la estructura valencial del núcleo verbal. De esta manera, podríamos, por un lado, tomar en consideración todas las características semánticas inherentes al núcleo verbal y, por otro, observar cómo estas características influyen en las diferentes interpretaciones modoaccionales que un mismo verbo puede o no poseer. Cabe destacar que este modo de análisis, asimismo, conllevaría consigo cambios terminológicos cuyas propuestas vamos a exponer a lo largo del trabajo.

Una vez estudiada la estructura valencial y conocidos los papeles temáticos del núcleo verbal, cabe observar cuáles son las características de los argumentos que vienen exigidos por éste último y que constituyen la proyección sintáctica de los papeles temáticos. Dicho de otro modo, se debe estudiar la naturaleza del CD y del sujeto para observar qué influencia ejercen en la interpretación modoaccional del verbo.

El último escalón de nuestra jerarquía corresponde a los CCT que, además de desempeñar un papel de detectores de las relaciones modoaccionales del predicado, asimismo, en ciertos casos, pueden imponer determinadas lecturas al predicado al que acompañan.

Por último, conviene precisar que vamos a analizar los verbos en formas infinitivas, en el caso de estudiar la estuctura valencial y la influencia de los CD y de los CCT; y en formas finitas, en el caso de estudiar las propiedades del sujeto.

Presentada la jerarquía de los factores, a continuación, vamos a examinar los ejemplos que fundamentan nuestro análisis, precedido éste por una breve explicación de los motivos que nos han hecho proponer este análisis jerárquico.

Al estudiar la clasificación cuatripartita de Vendler ${ }^{5}$ lo que llamó nuestra atención, en el primer lugar, ha sido el hecho de que esta clasificación abarque, al

${ }^{3}$ Cabe aclarar que el análisis modoaccional que proponemos en este trabajo surgió a base de unos ejemplos y todavía carece de investigación detallada, realizada a partir de un corpus más amplio.

${ }^{4}$ El análisis composicional de la Aktionsart de un predicado verbal no es nada novedoso. Muchos gramáticos, por ejemplo, De Miguel (op.cit., 2985-3008), B. Hlibowicka-Węglarz (op.cit., 45-58), cstán de acuerdo que la información modoaccional puede ser modificada por diferentes factores, como por ejemplo, la presencia de los complementos directos o adverbiales, por las características del sujeto o por la negación. En total, lo que nosotros consideramos indispensable, es aprovechar todas las informaciones aportadas por el entorno sintáctico-semántico para $\mathrm{cl}$ análisis modoaccional del verbo.

${ }^{5}$ Seguimos la clasificación presentada en el trabajo de Y. Morimoto (1998: 13-16). Esta clasificación abarca cuatro grupos de predicados de distinto contenido modoaccional: los estados, ejemplificado por el vcrbo saber; las actividades, por correr; las efectuaciones, por el predicado verbal correr cien metros y los logros, por llegar. En cuanto a la terminología, cabe mencionar que L.García Fernández (1998: 11), sustituye el término de las efectuaciones por las realizaciones. 
mismo tiempo, los verbos y los predicados verbales. En el segundo lugar, nos pareció curioso observar que en esta clasificación un mismo verbo puede pertenecer a diferentes subgrupos según le acompañe o no el complemento directo. Así, por ejemplo, el verbo comer pertenece al subgrupo de actividades y, en cambio, el predicado comer una manzana ya se adscribe al subgrupo de efectuaciones.

Según nosotros, este modo de clasificar, al incluir un mismo verbo en diferentes subgrupos, por una parte, puede generar confusiones a la hora de la clasificación y, por otra, en cierto modo, priva al verbo de su caracter primordial, dejando que un CD determine su interpretación modoaccional.

Además, quisiéramos llamar la atención al hecho de que la mayoría de los verbos incluidos en esta clasificación aparezcan sin complemento directo aunque, en algunos casos, la falta de éste puede provocar agramaticalidad. Por ejemplo, al subgrupo de los estados pertenece el núcleo verbal saber que, como es bien sabido, dentro de la oración exige un CD para su gramaticalidad. Así, resulta incorrecto decir:

* María sabe.

En cambio, si el verbo saber viene acompañado por un CD, la oración es totalmente gramatical:

María sabe inglés.

De modo que lo que proponemos para recuperar la primacía del verbo y, asimismo, evitar la clasificación de los puros núcleos verbales es recurrir a la estructura valencial del verbo y desde este nivel sintáctico-semántico describir todas las relaciones modoaccionales que el predicado engloba en su lectura, tomando en cuenta, al mismo tiempo, la influencia de los argumentos y los modificadores que le acompañan.

Ahora bien, vamos a describir el mecanismo de la determinación de relaciones modoaccionales de los predicados. Notemos que el verbo comer en su estructura valencial al seleccionar dos papeles temáticos, eso es, él de Agente y él de Tema, es bivalente. Conforme a ello, rige un sujeto y un $\mathrm{CD}$. Éste último, a su vez, al ser un CD afectado $^{6}$ goza de la posibilidad de aparecer implícita o explícitamente, influyendo así en el cambio de la interpretación modoaccional del predicado. Observémoslo en el siguiente esquema:

Estructura valencial:

Estructura argumental 1:

Ejemplo:

Subgrupo de la clasificación de Vendler:

Estructura argumental 2:

Ejemplo:

Subgrupo de la clasificación de Vendler: comer < Agente, Tema >

Sujeto comer $\mathrm{CD}$ explícito

comer una manzana

efectuaciones

Sujeto comer CD implícito

comer

actividades

${ }^{6}$ Sobre los argumentos o complementos directos afectados, con más detalle, trata, por cjemplo. Y. Morimoto (1998: 38-43). 
Gracias a estas características, en su estructura valencial el verbo comer es capaz de adquirir dos interpretaciones modoaccionales, perteneciendo así a dos subgrupos: a las efectuaciones y las actividades. En este lugar, quisiéramos proponer una nueva denominación para este tipo de predicados. Los llamaríamos verbos bimodoaccionales.

Como ya sabemos, el segundo factor que se debe incorporar en este análisis jerárquico son las características de los $\mathrm{CD}$. Hay que recordar que los $\mathrm{CD}$ delimitados imponen el carácter télico al verbo que los rige y los CD no delimitados, átelico. Por lo tanto, los primeros forman parte de las efectuaciones y los segundos de las actividades. Dicho esto, completemos nuestro esquema de arriba con la siguiente información:

Estructura argumental 1:

Ejemplo:

El subgrupo de la clasificación de Vendler:

Estructura argumental 2a:

Estructura argumental $2 \mathrm{~b}$ :

Ejemplo a:

Ejemplo b:

El subgrupo de la clasificación de Vendler:
Sujeto comer CD explícito delimitado comer una manzana efectuaciones

Sujeto comer CD implícito

Sujeto comer CD explícito no delimitado comer comer manzanas actividades

Asimismo, se puede observar la influencia del sujeto en la interpretación de la Aktionsart. Lo vamos a estudiar prestando y modificando un poco el ejemplo de E.de Miguel protagonizado por el verbo golpear ${ }^{7}$. Por nuestra parte, podemos agregar que el verbo golpear es bivalente y selecciona los papeles temáticos de Agente y de Finalidad. Vamos a determinar su lectura modoaccional, observando, esta vez, las características del sujeto. Fijémonos en las siguientes oraciones:

El viento golpea mi rostro

Sujeto (nombre cortinuo) golpear CD

actividades

El proyectil golpea la pared

Sujeto (nombre discontinuo) golpear CD

logros

Así, por seleccionar distintos tipos de sujeto el verbo golpear puede considerarse bimodoaccional.

El último factor que ayuda a reconocer las relaciones modoaccionales que un predicado puede poseer en su lectura es el CCT que, recordemos, por ser un elemento adicional, no entra en la estructura valencial del predicado. Sin embargo, su coaparición con un predicado no es casual. Como explica García Fernández (1998: 29), las expresiones temporales introducidas por durante y seguidas por un sintagma nominal cuantificado, como por ejempo, durante una hora, denotan el

\footnotetext{
${ }^{7}$ Las versiones originales de los ejemplos citados por la autora (op.cit., 3004) son las siguientes: El viento \{golpeó / golpeaba\} mi rostro. El proyectil \{golpeó / ?*golpeaba\} la pared.
} 
tiempo de la duración de un evento sin exigir la telicidad en el predicado y son compatibles con los predicados atélicos como estados y actividades. En cambio, las expresiones temporales introducidas por en y seguidas de un sintagma nominal cuantificado, como por ejemplo, en un segundo, en palabras del mismo gramático (1998: 11), "indican el tiempo que se tarda en efectuar por completo un evento" y coaparecen con realizaciones y logros. Podemos comprobarlo, aprovechando los ejempos arriba mencionados:

El viento golpea mi rostro durante una hora / *en un segundo

El proyectil golpea la pared en un segundo $/ *$ durante una hora

En estos ejemplos, podemos observar que los circunstantes de tiempo sirven para determinar la Aktionsart cuando el sujeto o los CD introducen cambios interpretativos. Sin embargo, hay casos cuando el CCT ayuda a reconocer diferentes interpretaciones modoaccionales en un predicado cuyo sujeto y $\mathrm{CD}$ no introducen susodichos cambios. Se trata, por ejemplo, del verbo conocer que, en su estructura valencial, selecciona Experimentante y Tema: $\langle$ Exp., $\mathrm{T}\rangle$.

Observemos cómo cambia su lectura al aparecer con distintos CCT:

conocer a alguien de toda la vida / desde que nació ${ }^{8}$. conocer a alguien a las tres de la tarde estados

logros

A modo de conclusión, quisiéramos señalar que la clasificación modoaccional de Vendler, en nuestra opinión, tiene caracter exterior al predicado verbal al dejar que un mismo verbo pertenezca a diferentes subgrupos modoaccionales según le acompañe o no un CD. Lo que nosotros proponemos en este trabajo, es reorientar la dirección del análisis modoaccional, centrándolo en la estructura interior del predicado verbal, o sea, su punto de partida sería la estructura valencial.

No obstante, al mismo tiempo, se debe tomar en consideración otros factores que influyen en la determinación de la Aktionsart del predicado verbal. Estos factores vienen representados, en primer lugar, por los argumentos verbales como el $\mathrm{CD}$ y el sujeto y, en el segundo lugar, por los elementos exteriores a la estructura argumental del predicado, esto es, por los CCT.

Desde tal perspectiva, las propiedades semánticas propuestas por Vendler pueden ser reagrupadas y reconsideradas como características uni- y bimodoaccionales, relacionadas con la estructura valencial de los predicados. A] concluir, a título de ejemplo, presentamos los verbos saber, cantar y morirse en cuyo caso se observa la correlación entre los rasgos en cuestión:

- $\underline{\text { saber }}$

saber : <Experimentante, Tema >

saberinglés estados

predicado unimodoaccional

${ }^{8}$ Desde y de son conjunciones tempo-aspectuales que en la clasificación propuesta por J. GarcíaMedall (1995: 229) pertenecen a la clase de la duratividad y se denominan conjunciones limitadoras de comienzo marcado. 
- cantar cantar una canción

cantar : < Agente, Tema>

cantar / cantar una canción

- morirse

morirse : $<$ Experimentante > morirse actividades

realizaciones

predicado bimodoaccional

logros

predicado unimodoaccional

\section{REFERERENCIAS BIBLIOGRÁFICAS}

Apresjan, D. J. (1974), Semantyka leksykalna. Synonimiczne środki jezyka. Wroclaw: Ossolineum, 2002.

García-Fernández, L. (1998), El aspecto gramatical en la conjugación. Madrid: Arco Libros.

García-Medall, J. (1996), Conjunciones temporales y aspecto. Moenia, vol. 1, 219-249.

Hlibowicka-Węg larz, B. (1998), Processos de expressāo do aspecto na língua portuguesa. Lublin:

Wydawnictwo Uniwersytetu Marii Curie Skłodowskiej.

Miguel, E. de (1999), El aspecto léxico, en I. Bosque y V. Demonte (eds), Gramática decriptiva de la

lengua española. Madrid: RAE, Espasa Calpe, v. 2, 2977-3059.

Mori moto, Y. (1998), El aspecto léxico: Delimitación. Madrid: Arco Libros.

- (2001), Los verbos de movimiento. Madrid: Visor Libros. 